\title{
Searches for neutrino fluxes in the EeV regime with the Pierre Auger Observatory
}

\section{Enrique Zas ${ }^{* a}$ for the Pierre Auger Collaboration ${ }^{b}$}

${ }^{a}$ Depto. Física de Partículas \& Instituto Galego de Física de Altas Enerxías, Universidade de Santiago de Compostela, 15782 Santiago de Compostela, Spain

${ }^{b}$ Observatorio Pierre Auger, Av. San Martín Norte 304, 5613 Malargüe, Argentina

E-mail: auger_spokespersons@fnal.gov

Full author list: http://www.auger.org/archive/authors_icrc_2017.html

The Pierre Auger Observatory has been used to search for neutrinos of energy exceeding 100 $\mathrm{PeV}$ by looking for inclined showers that develop deep in the atmosphere. Neutrinos of any flavor interacting deep in the atmosphere and triggering the Auger surface detector can be identified provided their zenith angles exceed 60 degrees. Also tau neutrinos that enter the Earth's crust with a zenith angle close to 90 degrees can interact and produce a tau that decays in the atmosphere inducing an "upward-going" shower that triggers the surface detector. The sensitivity obtained summing up these channels is shown to be comparable to other neutrino detectors in operation, and to constrain several models of cosmic-ray and neutrino production in the EeV region. The declination field of view for neutrino searches in these two channels spans from about -80 degrees to 60 degrees in equatorial coordinates. The Observatory has also been used for searches for neutrinos from point-like sources in the sky. We finally report on the results of the search for neutrino fluxes in coincidence with the gravitational wave events GW150914, GW151226 and GW170104 recently discovered with Advanced LIGO, and on their implications for the total energy emitted in EeV neutrinos by black hole merger events.

35th International Cosmic Ray Conference - ICRC2017

10-20 July, 2017

Bexco, Busan, Korea

\footnotetext{
* Speaker.
} 


\section{Introduction}

Neutrinos of $\sim 10^{18} \mathrm{eV}(1 \mathrm{EeV})$ are expected from interactions of Ultra-High Energy Cosmic Rays (UHECR) in the sources or during propagation through the Universe. Having no charge they point to their production sites even when they are shielded by large amounts of matter. Their energy spectrum is related to cosmic ray composition at the highest energies which is largely unknown [1]. As extraterrestrial neutrinos of several PeV have already been detected [2], extending the spectrum measurement to the $\mathrm{EeV}$ region is most natural to effectively constrain the production mechanisms of UHECR. Their detection would allow neutrino interactions to be probed at energies many orders of magnitude above those searched in accelerators and information about flavor ratios will further constrain the production mechanisms and may even signal new physics.

The Pierre Auger Observatory consists of a $3000 \mathrm{~km}^{2}$ array of water-Cherenkov detectors (SD) in a hexagonal grid of $1.5 \mathrm{~km}$ spacing to detect the particle front of air showers and a Fluorescence Detector (FD) that monitors the fluorescence emission of Nitrogen over this surface. It is located in Mendoza, Argentina, at an average latitude of $35.5^{\circ} \mathrm{S}$ and at a mean atmospheric depth (altitude) of $875 \mathrm{~g} \mathrm{~cm}^{-2}(1400 \mathrm{~m})$ [3]. It has been running and taking data since its construction started in 2004. The SD can be used to detect down-going EeV neutrino interactions in the atmosphere [4] (DG) and Earth-skimming [5] (ES) tau neutrinos. The interaction of nearly horizontal ES tau neutrinos in the Earth produces a tau lepton that can exit to the atmosphere and decay producing an air shower. This channel is most sensitive between $\sim 0.1$ and $25 \mathrm{EeV}$ where cosmological neutrinos (due to cosmic rays interactions with the Cosmic Microwave Background) are expected.

\section{Searching for UHE neutrinos at the Pierre Auger Observatory}

Atmospheric showers produced by inclined neutrinos (both ES and DG) that develop deep in the atmosphere can be distinguished from showers due to cosmic rays that interact in the upper layers. The signal of the SD stations is digitized in $25 \mathrm{~ns}$ time bins allowing a detailed measurement of the time profile of the shower front. In contrast, the electromagnetic component of inclined cosmic ray showers gets absorbed and only the muon component reaches ground level. These showers, that are regularly detected and reconstructed [6], consist of $\sim 20$ to 200-GeV energy muons that travel nearly parallel to the shower axis and undergo few interactions. As a result they accumulate small time delays and deposit the energy in a short time, while deep neutrino showers produce signals with a much broader time spread. This provides the basis for neutrino identification [4]. As the zenith angle rises it becomes easier to separate the neutrino candidates because the total slant depth of the atmosphere increases making the differences more patent.

The searches for ES and DG events require independent procedures for event selection, neutrino identification and exposure calculation. Extensive simulations of neutrino events are made varying the energy, zenith and azimuth angles, depth of interaction and impact parameter of the neutrino and, in the ES case, the tau lepton decay altitude. The response of the SD is also simulated with standard software. The search strategy involves a pre-selection of inclined events followed by a selection of neutrino candidates based on a single optimized variable. A cut value is decided a priori comparing the distributions of this variable for simulated neutrino events to that of a selected background of cosmic ray showers, taken from a small fraction of the data. Simulated neutrino 
events and background are treated with the same selection and identification procedures. The efficiency of the neutrino search, needed to calculate the exposure, is obtained as the ratio of neutrino candidates to the total number of simulated neutrinos as a function of the relevant variables.

For optimization purposes inclined data are searched in three different zenith angle ranges. (1) Earth skimming tau neutrinos (ES) can be detected only with zenith angles between $90^{\circ}$ and $95^{\circ}$. The ES selection requires a minimum of three stations, a high eccentricity of the elliptic shape of the triggered area on the ground and an apparent speed of the trigger times between station pairs with an average value very close to $c$ and a small spread. (2) The selection of DG neutrinos between $75^{\circ}$ and $90^{\circ}$ (DGH) is made for events with at least 4 stations using the same variables as the ES selection with less stringent cuts. In addition the zenith angle of a plane fit must exceed $75^{\circ}$. (3) To select DG neutrinos between $60^{\circ}$ and $75^{\circ}$ (DGL) the reconstructed zenith from a plane front fit is required to be in the $58.5^{\circ}-76.5^{\circ}$ range to allow for reconstruction tolerances in the simulated neutrino events (see [7] for full details). .

To separate neutrinos from cosmic ray showers, observables have been chosen related to the width of the signal trace, particularly the Area over Peak (AoP) of the digitized signal. For the ES selection the average AoP of the triggered stations $(<\mathrm{AoP}>)$ is chosen as a discrimination variable [7]. For the DG cases a Fisher discriminant approach is used combining up to 10 variables using the AoP of 4 (4 or 5) early (central) stations in the DGH (DGL) selections [7]. The critical value of the discrimination variable (above which events are classified as potential neutrino candidates) is chosen in each case using the distribution of background events, obtained from a small fraction of the data, assumed to be overwhelmingly (if not totally) made up of cosmic ray showers. These distributions have an exponential tail which can be easily extrapolated to obtain the parameter value corresponding to a background rate of one event every 50 years for the full SD array. A further subdivision based on the zenith angle (number of triggered SD stations) is made in the DGL (DGH) selection to select the cut in the corresponding discriminant variable. The AoP distribution for ES events is shown in Fig. 1. No neutrino candidates have been found.
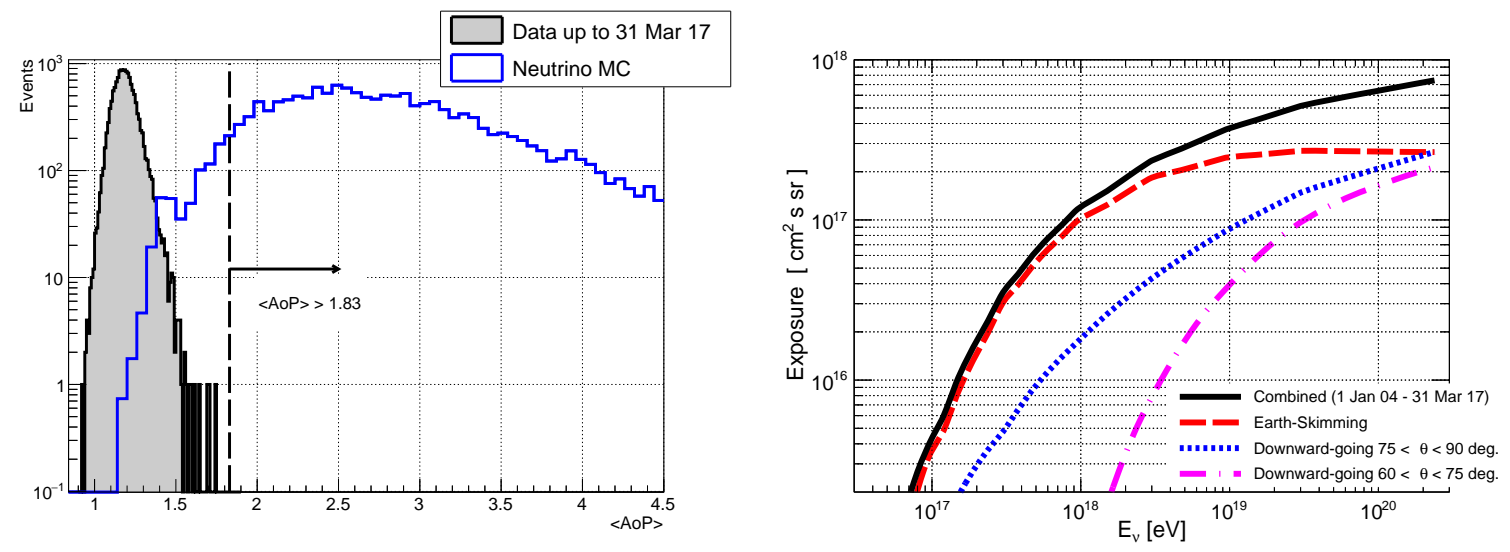

Figure 1: Left: Distributions of $\langle$ AoP $\rangle$ after ES selection. The shaded histogram is the data up to 31 Mar 2017 (excluding the data used to select the cut) and the empty blue histogram are simulated ES $v_{\tau}$ events. The vertical line represents the cut on $\langle$ AoP $\rangle>1.83$ to search for candidates.

Right: Total exposure (solid) as a function of neutrino energy and contributions of the ES (dashes), DGH (dots) and DGL (dot dashes) searches at Earth assuming equal fluxes for all neutrino flavors. 


\section{Exposure calculation and neutrino bounds}

To obtain a bound on the diffuse flux, the integral of the interaction and detection probabilities over surface area, solid angle and time is calculated as a function of neutrino energy. This quantity is the effective exposure which, convolved with a given neutrino flux spectrum, gives the expected number of events.

For ES tau neutrinos the probability of the tau lepton exiting the Earth is calculated as a function of arrival direction and tau energy using a propagation code for the tau neutrinos in rock that accounts for neutral and charged current interactions and includes regeneration. This probability is convolved with two others, that of the tau decaying which is a standard exponential and that of triggering, selecting and identifying the neutrinos which is obtained from simulations. Both depend on the decay position of the tau lepton. The exposure is obtained integrating over area, arrival direction, time and distance to the decay. For the DG case the calculation just involves an integral over area, arrival direction, interaction depth and time of the probabilities for interaction and for the trigger, selection and identification. Different channels must be considered depending on neutrino flavor and interaction type because the energy transfers to the shower are different. Three calculations are required: neutral current interactions, charged current electron neutrino interactions and charged current tau neutrino interactions. Charged current interactions of muon neutrinos are treated identically to neutral currents of all flavor neutrinos concerning the energy transfer, since the atmospheric shower is induced by the nuclear fragments.

The data set that has been used for all searches presented here spans the period from 1 Jan 2004 to 31 March 2017. A small fraction of the data is excluded because it corresponds to unusual periods in which the array was unstable. This period includes the un-blinding of nearly four years of data compared to previous results [7]. To account for the changing of array configuration particularly throughout the construction phase, the time integral is performed sampling the effective probabilities over real array configurations chosen every three days. As the area over which the neutrino flux is simulated always exceeds that of the array, the procedure also accounts for edge effects and occasional missing stations. The results of the exposure calculations for the ES, DGH and DGL searches and the combined exposure assuming all neutrino flavors have the same weight are displayed in Fig. 1. The figure illustrates that the Earth-skimming neutrinos dominate the exposure in spite of the reduced solid angle to which the detector is sensitive.

\subsection{Limits to diffuse fluxes of UHE neutrinos}

In the Feldman-Cousins approach if no events are observed the 90\% C.L. limit is set for a flux predicting 2.39 events. The limits for a diffuse isotropic flux of single flavor neutrinos are shown in Fig. 2 using a semi-Bayesian extension of this approach to account for the systematic uncertainties. Two types of bounds are calculated: An integral bound is obtained integrating a conventional $k E^{-2}$ neutrino spectrum, giving $k<510^{-9} \mathrm{GeVcm}^{-2} \mathrm{~s}^{-1}$. It is conventionally plotted from 0.1 to 25 $\mathrm{EeV}$, corresponding to the range in which $90 \%$ of the events are expected. A differential bound is calculated integrating the neutrino flux over consecutive energy bins of 0.5 in $\log _{10} E_{v}$. It clearly indicates that the SD is particularly well suited for detecting cosmological neutrinos resulting from interactions of UHE protons with the Cosmic Microwave Background. The obtained limits are compared to those from current neutrino telescopes in the equal flavor scenario. 


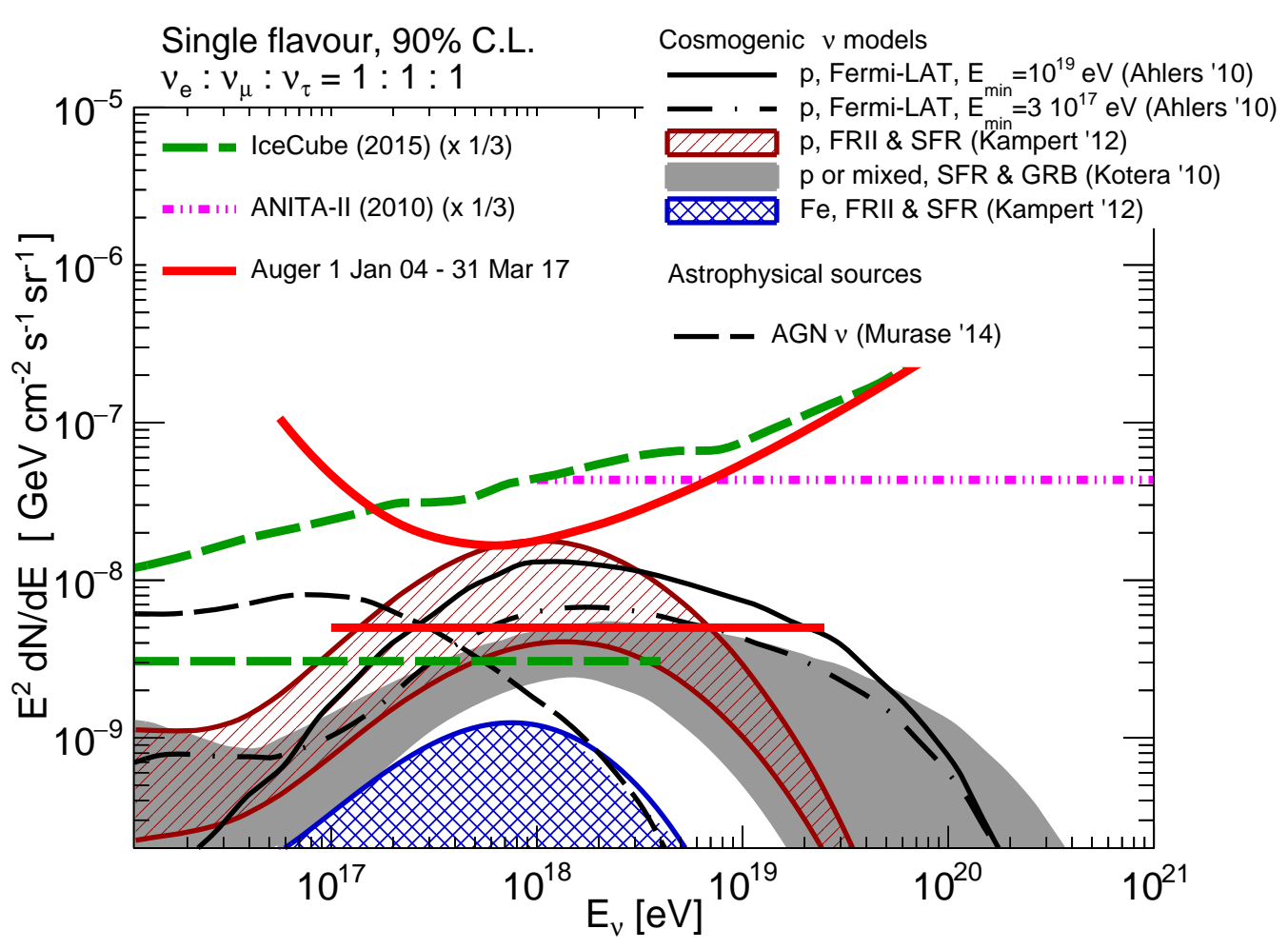

Figure 2: Integral upper limit (at $90 \%$ C.L.) for a diffuse neutrino flux of UHE $d N / d E_{v}=k E^{-2}$ given as a normalization, $k$, (straight red line), and differential upper limit (see text). Limits are quoted for a single flavor assuming equal flavor ratios. Similar limits from ANITAII [8] and IceCube [9] are displayed along with prediction for several neutrino models (cosmogenic [10,11, 12], astrophysical [13].)

\subsection{Limits to point-like sources of UHE neutrinos}

The Earth-skimming channel is very effective at converting the tau neutrinos into exiting tau leptons when the arrival direction is very close to the horizontal. It can be shown that over $90 \%$ $(\sim 100 \%)$ of the ES exposure is obtained for zenith angles between $90^{\circ}$ and $92.5^{\circ}\left(95^{\circ}\right)$. As a result the sky coverage provided by these interactions reaches declinations between $-54.5^{\circ}$ and $59.5^{\circ}$. The DG selections enhance the visible declination band towards the south all the way to $-84.5^{\circ}$ covering a large fraction of the sky. The exposure as a function of zenith can be converted to an average exposure for a given declination integrating in right ascension. It displays strong peaks for the ES selection close to two extreme declinations apparent in the obtained bounds.

The non-observation of neutrino candidates is cast into a bound on point sources which is calculated as a function of declination, $\delta$, also assuming a flavor ratio of $1: 1: 1$. The results are displayed in Fig. 3, for the first time combining the three searches and for data that have an increase of about seven years of full exposure over previous results [15].

\subsection{Targeted searches for correlations with the GW events}

The reported detection of gravitational wave events produced by bynary Black Hole $(\mathrm{BH})$ mergers by the Advanced Ligo Collaboration has triggered a targeted search for coincidence events that would complement these observations. BH mergers could accelerate cosmic rays to the high- 

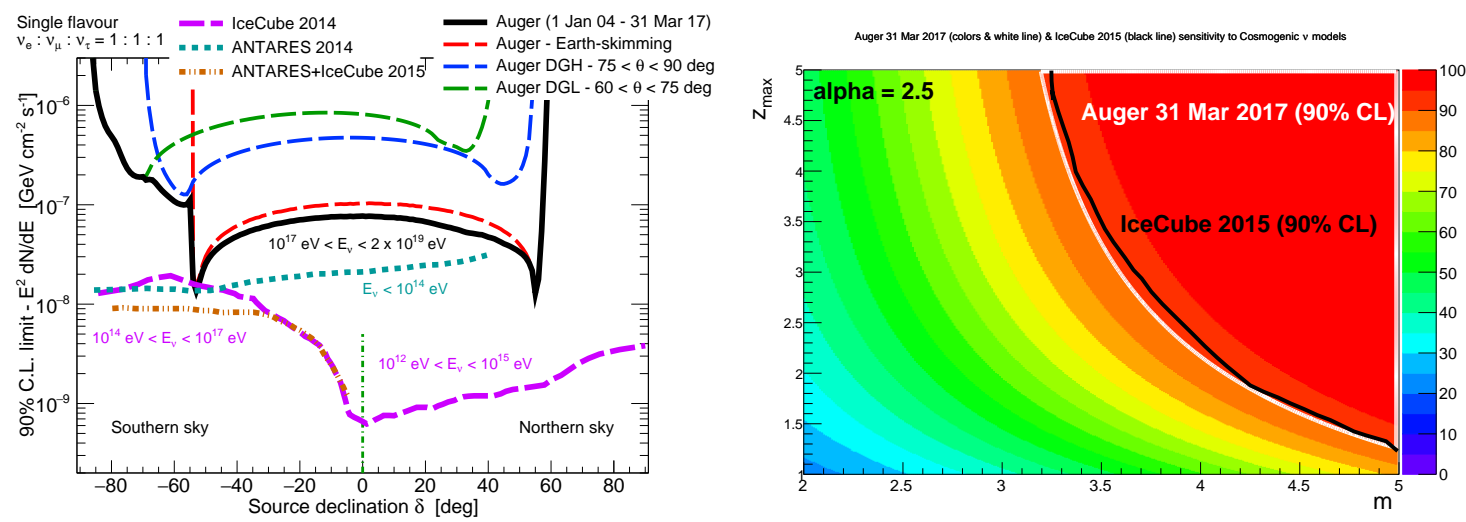

Figure 3: Left: Upper limits at $90 \%$ C.L. for $k^{\mathrm{PS}}$ as a function of the source declination for ES, DGH and DGL searches as labeled (assuming a single flavor point-like flux of UHE neutrinos $d N / d E_{v}=k^{\mathrm{PS}} E_{v}^{-2}$ ). Also shown are the sensitivities for IceCube [16], ANTARES [17] and a combination of both [18]. Note the different energy ranges.

Right: Constraints on parameter space for cosmological neutrinos in proton models (assuming a powerlaw $d N / d E \propto E^{-2.5}$ ) as a function of $m$ (source evolution) and $z_{\max }$ (maximum redshift of the sources) following [22]. Colored areas represent different confidence levels of exclusion. The region above the black (white) line is excluded at 90\% C.L. by IceCube [9] (Auger) data.

est energies and produce neutrinos provided there are magnetic fields and disk debris from the progenitor stars [19]. A search has been made for EeV events in the data collected with the Observatory and correlated in time and position with events GW150914, GW151226, GW170104 and the candidate event LVT151012. In each case two time windows have been considered to search for coincident neutrino events. One is of $\pm 500 \mathrm{~s}$ about detection time, motivated by an upper limit to the duration of the prompt phase of Gamma Ray Bursts (GRB) and the second one a single day after the event, motivated by an upper limit to the duration of GRB afterglows [20].

The directionality of the neutrino exposure makes the effectivity of the search quite dependent on the event position. Since this position is only known as a broad region of hundreds of square degrees, the results are given as a function of the event declination. The calculation of the exposure for each class of events is similar to that obtained for point source searches but the time integral is now limited to the preselected search window. It is only accounted for to the extent the source is visible in each one of the three zenith angle ranges considered.

Because of the zenith angle restrictions the visible part of the sky is limited at any instant as shown in Fig. 4 left. When we consider the short time-window, the 90\% C.L. position of GW150914 has only a marginal overlap with the corresponding sky coverage of the Observatory for the DGH selection. A resulting bound could only be obtained if the position of the event was known to be in the overlapping region. The situation with GW151226, GW170104 and LVT151012 is different since there is a substantial overlap between the sky coverage with both with ES and DG searches and the reported positions, particularly for GW151226 (see Fig 4). No inclined background showers from cosmic rays were actually observed at all during the 1000 s-wide window around any of the GW events.

For the one-day period the instantaneous exposure is averaged over a sidereal day GW150914, 

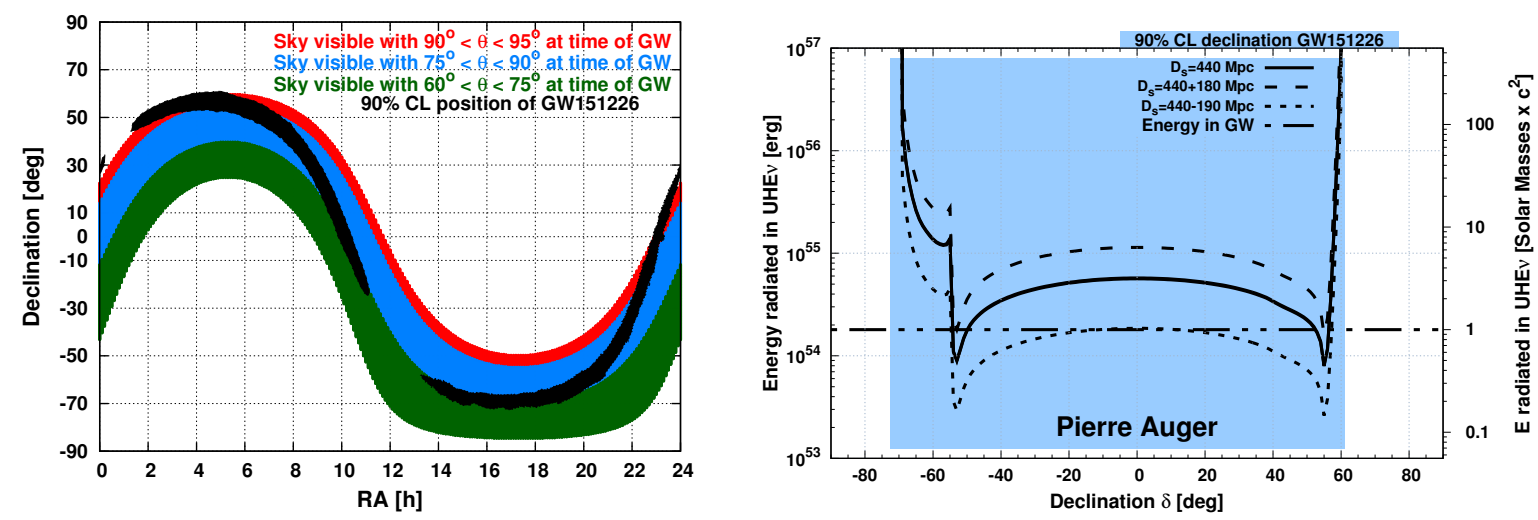

Figure 4: Left: Instantaneous sky coverage of the different selections as labeled at the time of the detection of GW151226. The black contour region corresponds to the $90 \%$ C.L. region for the GW event location. Right: Constraints on the radiated energy in UHE neutrinos (per flavor) from the source of GW151226 as a function of $\delta$ [20]. Energies above the solid line are excluded at the 90\% C.L.. The calculation is done for a distance $D_{s}=410 \mathrm{Mpc}$ (dashed lines correspond to reported distances at the $90 \%$ C.L.). The dot-dashed horizontal line represents $E_{\mathrm{GW}} \simeq 1.9 \times 10^{54} \mathrm{erg}$, the inferred energy radiated in gravitational waves for that event [21]. The shaded region indicates the $90 \%$ C.L. region for the GW event location.

GW170104 and LVT151012 have large overlap with the sky coverage of the Observatory and for GW151226 practically all the $90 \%$ C.L. declination band of the event is covered as illustrated by the shadowed band in Fig. 4. Inclined showers were observed within a day of the detected events but these were completely consistent with those expected from UHE cosmic rays.

A bound can be obtained assuming an $E^{-2}$ spectrum. The $90 \%$ C.L. upper bound to the flux is obtained by fixing the normalization to expect 2.39 events. A bound can also be obtained on the total energy radiated in neutrinos using the luminosity distances quoted for the events, illustrated in Fig. 4 for GW151226. Bounds for GW150914 are better in relation to the GW energy radiated [20] while for GW170104 they are weaker because the event is located further away [21].

\section{Summary and Discussion}

The Auger Observatory is effective at searching for neutrinos of energies exceeding $0.1 \mathrm{EeV}$ selecting inclined showers that have significant electromagnetic component. The search for neutrinos has given differential and integrated (assuming an $E^{-2}$ spectrum) bounds to diffuse and point source fluxes using data from 1 Jan 2004 to 31 March 2017 and assuming equal flavor ratios. Precise statements about given models require folding the exposure with the model spectrum to obtain the expected number of events. Different models for cosmogenic neutrinos that attempt to explain the origin of cosmic rays are excluded at the $90 \%$ C.L., particularly those that assume proton primaries and attribute a strong evolution with redshift to the sources comparable to that of FanaroffRiley type II (FRII) galaxies $[12,11]$. Some models that normalize the proton flux to the diffuse GeV gamma-ray flux measured by FERMI-LAT [10] are also excluded. A model that assumes protons with moderate evolution close to that of SFR [14] is disfavored while others [12, 11, 14] assuming heavier primaries would need a significant increase in exposure to become excluded. 
Some astrophysical models are also excluded at $90 \%$ C.L. for instance that assuming cosmic ray acceleration in radio-loud AGN [13].

It is possible to use a conservative analytical approximation of the cosmogenic neutrino flux so that exclusion plots can be made as functions of the most relevant parameters, namely $m$, the source evolution, $z_{\max }$, the maximum redshift to which the cosmic ray production is integrated, $\alpha$, the spectral index, and $E_{\max }$, and the maximum energy of the cosmic ray flux [22, 23]. The corresponding plot for a spectral index of 2.5 and for $E_{\max }=300 \mathrm{EeV}$ is shown in Fig. 3.

Finally new limits on steady point sources have also been obtained and a targeted search for correlations with the gravitational wave events from Black Hole mergers have yielded limits on a possible UHE neutrino emission and on its energy budget. These limits help to constrain all kinds of models as part of a multi-messenger approach.

\section{References}

[1] D. Martello for the Pierre Auger Collaboration; these proceedings.

[2] M. G. Aartsen et al. Phys. Rev. Lett. 113, 101101 (2014).

[3] A. Aab et al. Nuc. Inst. Meth. A789, 172 (2015).

[4] K. S. Capelle et al.; Astropart. Phys. 8, 321 (1998).

[5] X. Bertou et al.; Astropart. Phys. 17, 183 (2002).

[6] A. Aab et al.; JCAP 1408, no. 08, 019 (2014).

[7] A. Aab et al. [Pierre Auger Collab.], Phys. Rev. D 91, 092008 (2015).

[8] P. W. Gorham et al. [ANITA Collab.], Phys. Rev. D 85, 049901(E) (2012).

[9] M. G. Aartsen et al. [IceCube Collab.] Phys. Rev. Lett. 117, 241101 (2016).

[10] M. Ahlers et al., Astropart. Phys. 34, 106 (2010).

[11] K. -H. Kampert, M. Unger, Astropart. Phys. 35, 660 (2012).

[12] D. Allard et al., JCAP 10, 013 (2010).

[13] K. Murase, Y. Inoue, and C. D. Dermer, Phys. Rev. D 90, 023007 (2014).

[14] R. Aloisio, et al., JCAP 1510, no. 10, 006 (2015).

[15] P. Abreu et al., Astrophys. J. 755, L4 (2012).

[16] M.G. Aarsten et al., Astrophys.J. 796, 109 (2014).

[17] S. Adrian-Martinez et al. [ANTARES Collab.], Astrophys. J. Lett. 786, L5 (2014).

[18] S. Adrian-Martinez et al., Astrophys. J. 823, 65 (2016).

[19] K. Kotera and J. Silk; Astrophys. J. 823, no. 2, L29 (2016).

[20] A. Aab et al. [Pierre Auger Collab.], Phys. Rev. D 94, 122007 (2016).

[21] B. P. Abbott et al. [LIGO Scientific Collab. and Virgo Collab.], Phys. Rev. Lett. 116, 061102 (2016); ibid. 116241103 (2016); ibid. 118221101 (2017).

[22] S. Yoshida and A. Ishihara, Phys. Rev. D 85, 063002 (2012).

[23] M. G. Aartsen et al. Phys. Rev. Lett. 117, 241101 (2016). 